\title{
Decrystallization of cellulose under the influence of elastomer-assisted mechanical and mechanochemical shear
}

\author{
R LAVANYA and N NATCHIMUTHU* D \\ Department of Rubber and Plastics Technology, Madras Institute of Technology Campus, Anna University, Chromepet, \\ Chennai 600 044, India \\ *Author for correspondence (nmuthu@ mitindia.edu)
}

MS received 4 November 2017; accepted 19 January 2019; published online 23 May 2019

\begin{abstract}
Microcrystalline cellulose (MCC) was subjected to mechanical and mechanochemical shear in a rubber mixing milling. Nitrile rubber (NBR) and ethylene-propylene-diene monomer (EPDM) rubber were used to induce shear on cellulose during treatment. Solid-state interactions between $N, N^{\prime}$-dimethylacetamide/lithium chloride $(\mathrm{DMAc} / \mathrm{LiCl})$ and cellulose particles were facilitated during mechanochemical shear. Fourier transform infrared spectroscopy, X-ray diffraction analysis, thermogravimetric analysis, scanning electron microscopy and swelling studies were carried out on MCC, DMAc/LiCl-treated MCC, MCC subjected to mechanical shear and DMAc/LiCl-treated MCC subjected to mechanochemical shear. Crystallinity and swelling behaviour of the untreated and merely DMAc/LiCl-treated MCC samples, in the absence of any shear, were found to be similar. However, when MCC was subjected to mechanical shear assisted by $\mathrm{NBR}$ and $\mathrm{DMAc} / \mathrm{LiCl}$-treated $\mathrm{MCC}$ subjected to mechanochemical shear assisted by EPDM, the resultant MCC samples exhibited significant reduction in their crystalline index with increased swelling. When DMAc/LiCl-treated MCC was subjected to mechanochemical shear assisted by NBR and mechanical shear alone on MCC, assisted by EPDM, did not exhibit any appreciable change in the crystalline index and swelling behaviour of the resultant MCC samples. While NBR-assisted mechanical shear was found to reduce crystallinity in untreated MCC, crystallinity in DMAc/LiCl-treated MCC was found to be reduced significantly with EPDM rubber-assisted mechanochemical shear.
\end{abstract}

Keywords. Microcrystalline cellulose; DMAc/LiCl; crystallinity; elastomer; dissolution.

\section{Introduction}

Cellulose is the most abundant material available in various forms of biomass. The properties of cellulose are strongly influenced due to the presence of hydroxyl groups and their arrangement in cellulose molecules. These hydroxyl groups form very strong inter- and intra-molecular hydrogen bonds between cellulose chains, thereby rendering it to be highly crystalline [1]. An extensive network of hydrogen bonds and the resultant crystallinity make cellulose dissolution very difficult in organic solvents. Acidic or alkaline aqueous solutions are capable of dissolving cellulose effectively and employed commercially for the manufacture of derivatives, such as cellulose nitrate, cellulose acetate and regenerated cellulose. However, even highly polar organic solvents are unable to break the hydrogen bonds in cellulose and unable to make the latter, soluble in the former. This insoluble nature of cellulose in organic solvents is, therefore, a severe limitation to carry out further chemical modifications. However, complex ionic liquid systems are found to be useful for dissolving cellulose and form solutions. For examples, 1-alkyl-3-methylimidazolium chloride $\left[\mathrm{C}_{n}\right.$ mim] $][\mathrm{Cl}](n=4-8)$ ionic liquids are used to dissolve cellulose [2-7]. When dissolved in ionic liquids, ions such as chloride and acetate anions are found to replace the $\mathrm{OH}-\mathrm{O}$ hydrogen bonds in cellulose chains with the $\mathrm{OH}-\mathrm{Cl}^{-} \mathrm{OH}$ and $\mathrm{OH}-\mathrm{COO}^{-}$hydrogen bonds. The $N, N^{\prime}-$ dimethylacetamide/lithium chloride ( $\mathrm{DMAc} / \mathrm{LiCl})$ solvent system has also been found to be effective in dissolving cellulose [8-12].

Application of mechanical forces to reduce the crystalline structure of cellulose to increase its solubility and enhance the accessibility of hydroxyl groups for chemical modification has been reported. When cellulose is subjected to dry mechanical activation in a ball mill it has exhibited a significant increase in alkaline solubility and a drop in its degree of polymerization [13]. Mechanical milling carried out on cellulose in a pan mill, has been found to increase the surface area and alkaline solubility, while reducing its crystalline index and particle size. Such mechanical actions are reported to play a pivotal role in reducing hydrogen bonds, thermal stability, activation energy and degree of crystallinity in cellulose, thereby enhancing its solubility $[14,15]$. Compressive and shear stresses during mechanical actions on cellulose are reported to defibrillate it extensively as the milling cycles are increased [16]. Such defibrillated samples disperse very well when melt blended with polymers such as poly(vinyl alcohol) 
in the presence of plasticizers and produce eco-composites with improved properties, such as tensile strength, elongation at break and modulus.

The fibrous nature combined with its abundant availability makes cellulose one of the widely used reinforcing materials in making many composites. It is often used in rubber formulations as the reinforcing filler as an alternative to carbon black. Cellulose short fibres [17-21] and cellulose nanocrystals [22-25] are used as fillers in many rubber composites. Since the structure of cellulose molecules is inherently stiff and rigid, when properly dispersed in the rubber matrix, the resultant composites can have a range of properties depending on the aspect ratio of cellulose fibres, fibre orientation, fibrematrix adhesion and the type of rubber. Structural changes induced in cellulose molecules, while making such composites, however, are less understood especially when they are subjected to intensive shear stresses during mixing and compounding. In an earlier investigation, the authors have reported morphological changes in $\mathrm{DMAc} / \mathrm{LiCl}$ pre-treated cellulose powder subjected to mechanochemical shear using natural rubber as the shear transfer medium [26]. A combination of shear and $\mathrm{DMAc} / \mathrm{LiCl}$ pre-treatment of cellulose has been found to synergistically reduce the crystalline index in cellulose which enhanced its swelling. To transmit shear forces effectively onto cellulose, elastomers that do not show much change in their viscosity at ambient temperature could be considered for shear-induced mechanochemical modifications in cellulose.

The present work is focussed on to study the morphological and structural changes in microcrystalline cellulose (MCC) when subjected to mechanical and mechanochemical shear using nitrile rubber (NBR) and ethylene-propylene-diene monomer (EPDM) rubber. These rubbers have the dual function of serving as carriers and also as shear transfer media for $\mathrm{MCC}$ and DMAc/LiCl pre-treated MCC samples. Changes in the structure and morphology of mechanochemically-treated MCC samples are characterized using Fourier transform infrared spectroscopy (FTIR), X-ray diffraction (XRD), thermogravimetric analysis (TGA), scanning electron microscopy (SEM) and swelling studies and the results are compared with those of cellulose samples subjected to elastomer-assisted mechanical shear.

\section{Experimental}

\subsection{Materials and methods}

MCC (CAS no. 9004-34-6) was purchased from Sigma Aldrich-India and used after drying at $120^{\circ} \mathrm{C}$ for $1 \mathrm{~h}$ in an air circulating oven. DMAc, LiCl, ethyl methyl ketone (MEK) and toluene, all laboratory grades, were obtained from Sisco Research Laboratories Pvt. Ltd., India, and used as received. NBR-KNB35L (34\% acrylonitrile content) and EPDM rubber (a terpolymer of ethylene-propylene-diene monomer with an ethylidene norbornene content of $4.5 \%$ ) were supplied by RK Polymers, India, and used as received.

\subsection{Mechanochemical treatment of MCC}

A two-roll rubber mixing mill with a friction ratio of $1: 1.4$ was used in this study. The two rolls run at different speeds and exert intensive shear forces on cellulose through the rubber as the carrier and shear transfer medium. Ten millilitres of $9 \% \mathrm{DMAc} / \mathrm{LiCl}$ solution was added to $50 \mathrm{~g}$ of $\mathrm{MCC}$ and tumble mixed for $30 \mathrm{~min}$ in a closed round bottom flask and the contents were kept for $24 \mathrm{~h}$, so that MCC completely absorbed the DMAc/LiCl solution. The DMAc/LiCl-treated MCC sample thus obtained was used in the mechanochemical treatment. Fifty grams of the chosen rubber was made into a smooth sheet on the mill for 2 min. While the rubber sheet was in the form of a continuous band over the roll, $50 \mathrm{~g}$ of the DMAc/LiCl-treated MCC sample was slowly incorporated into rubber and subjected to mechanochemical action for 15 min. Close proximity between the absorbed DMAc/LiCl solvent and MCC under the influence of mechanical shear with enhanced solid-state interactions between them constituted the mechanochemical treatment. The mill rolls were kept at room temperature by circulating cold water to prevent any significant increase in temperature.

After the mechanochemical treatment, the rubber-MCC sheet was removed from the mill and kept for $24 \mathrm{~h}$. Mechanochemically treated MCC was separated from the rubber-MCC sheet by selectively dissolving the rubber matrix using an appropriate solvent. While MEK was used as the solvent to separate treated-MCC from NBR, toluene was used to separate it from EPDM rubber. Since the rubbers were dissolved in their respective solvents, the mechanochemically treated MCC particles settled down and were separated after decanting the rubber solution. The cellulose samples thus obtained were washed repeatedly five times using the respective rubber solvents to ensure the complete removal of rubber fractions and were finally dried at $80^{\circ} \mathrm{C}$ in an air circulating oven for $24 \mathrm{~h}$ and stored after cooling to room temperature. For comparison, untreated MCC samples (without DMAc/LiCl treatment) were also subjected to mechanical shear using the rubbers and the samples were separated from the rubber sheets by dissolving the rubbers in their respective solvents as described above. The details of the mechanical and mechanochemical treatments are presented in table 1.

\section{Characterization}

Samples of untreated MCC, DMAc/LiCl-treated MCC, untreated MCC subjected to mechanical shear and DMAc/LiCl-treated MCC subjected to mechanochemical shear were characterized using FTIR, XRD, TGA and swelling behaviour to understand the effects of mechanical and mechanochemical treatments on hydrogen bonding, crystallinity and thermal stability of cellulose. Regenerated 
Table 1. Crystalline index of MCC samples subjected to elastomer-assisted mechanical and mechanochemical shear.

\begin{tabular}{lcc}
\hline Sample & Process & Crystalline index (CI) (\%) \\
\hline MCC & No shear & 74.2 \\
DMAc/LiCI-MCC & No shear & 73.8 \\
MCC & NBR-assisted mechanical shear & 66 \\
DMAc/LiCI-MCC & NBR-assisted mechanochemical shear & 73.5 \\
MCC & EPDM rubber-assisted mechanical shear & 75.3 \\
DMAc/LiCI-MCC & EPDM rubber-assisted mechanochemical shear & 64 \\
\hline
\end{tabular}

cellulose samples obtained at the end of $48 \mathrm{~h}$ of swelling were analysed using SEM.

\section{$3.1 \quad F T I R$}

Untreated MCC, DMAc/LiCl-treated MCC and MCC samples obtained from rubber-MCC sheets after mechanical and mechanochemical treatments were used for FTIR analysis. Samples were mixed with $\mathrm{KBr}$ powder to produce pellets and spectra were recorded using a Perkin Elmer spectrometer (USA) in the range of $500-4500 \mathrm{~cm}^{-1}$.

\section{$3.2 X R D$}

XRD analysis was performed using a Bruker Kappa APE XII diffractometer in the $2 \theta$ range between 10 and $50^{\circ}$ at a scan step of $0.05^{\circ}$. The degree of crystallinity, expressed as the crystalline index ( $\mathrm{CrI} \%)$, was calculated using the following equation described by Segal [27].

$$
\operatorname{CrI} \%=\frac{I_{002}-I_{\mathrm{am}}}{I_{002}} \times 100
$$

where $I_{002}$ and $I_{\mathrm{am}}$ are the peak intensities at $2 \theta$ values of 22 and $18^{\circ}$ representing crystalline and amorphous parts of cellulose, respectively.

\section{$3.3 \quad T G A$}

Thermal stabilities of untreated MCC and MCC samples subjected to mechanical and mechanochemical treatments were investigated using a thermogravimetric analyser (TA Instruments, TGA model Q-50). Weight loss measurements were carried out from room temperature to $400^{\circ} \mathrm{C}$ at a heating rate

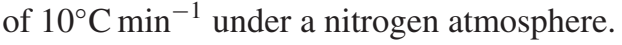

\subsection{Swelling studies}

Swelling and dissolution behaviours of mechanically and mechanochemically treated MCC samples were compared with that of untreated MCC and DMAc/LiCl-treated MCC. One gram of MCC from each sample was placed separately in glass bottles containing $10 \mathrm{ml}$ of $\mathrm{DMAc} / \mathrm{LiCl}$ as the solvent for swelling. After $48 \mathrm{~h}$ of swelling, the extent of swelling and/or dissolution was visually observed and photographs of the samples were taken using a digital camera.

\subsection{Surface morphological studies of regenerated cellulose}

After $48 \mathrm{~h}$ of swelling, cellulose samples from the DMAc/LiCl solvent were regenerated and separated by washing repeatedly with distilled water and dried at $80^{\circ} \mathrm{C}$ for $24 \mathrm{~h}$. Dried samples thus obtained were sputter-coated with gold and used for SEM analysis using an FEI Quanta FEG 200 model SEM to understand the effects of mechanical and mechanochemical shear on the surface morphological features of MCC in their regenerated state.

\section{Results and discussion}

\subsection{FTIR data of cellulose samples subjected to mechanical and mechanochemical shear}

FTIR spectroscopy has been used to understand the intermolecular and intramolecular hydrogen bonds in cellulose. The change in hydroxyl stretching vibrations of samples subjected to mechanical and mechanochemical shear assisted by NBR is shown in figure 1. Mechanical and mechanochemical treatments can alter the nature of hydrogen bonds between hydroxyl groups present in cellulose due to extensive compressive and shear forces exerted on the samples. Altered hydrogen bonds manifest in the infrared absorption peak positions of the hydroxyl groups. The broad $\mathrm{OH}$ stretching peaks at $3400 \mathrm{~cm}^{-1}$, shown in figure 1a and $\mathrm{b}$, comprise intra- and inter-molecular hydrogen bonds in untreated and DMAc/LiCl-treated MCC. Identical peaks at 3400 and $3392 \mathrm{~cm}^{-1}$ indicate that merely treating MCC with $\mathrm{DMAc} / \mathrm{LiCl}$ does not have any significant effect on the intermolecular hydrogen bonds. However, when untreated MCC is subjected to shear in the presence of NBR (figure 1c), the intermolecular hydrogen bonds are seen to be disrupted and shifted from 3400 to $3351 \mathrm{~cm}^{-1}$ [28]. Disruption of intermolecular hydrogen bonds is associated with an increase in intramolecular hydrogen bonds for which the absorption occurs at $3351 \mathrm{~cm}^{-1}$ [29,30]. Under the influence of shear, strong polar interactions between $\mathrm{OH}$ groups in 


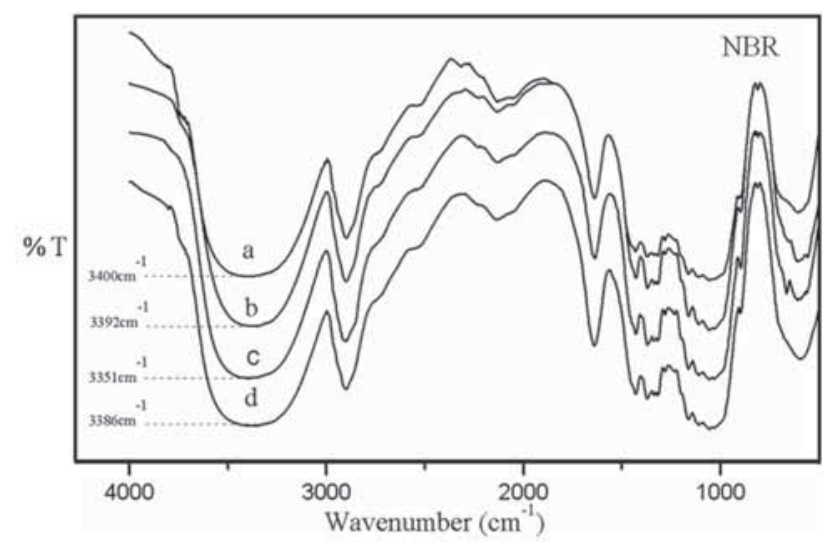

Figure 1. FTIR spectra of (a) untreated MCC, (b) DMAc/LiCltreated MCC, (c) untreated MCC subjected to NBR-assisted mechanical shear and (d) DMAc/LiCl-treated MCC subjected to mechanochemical shear.

cellulose and CN groups of NBR could be attributed for the disruption of intermolecular hydrogen bonds. The partial negative charge of the nitrogen atom in the nitrile group can interact with the hydrogen of cellulose hydroxyl groups to form hydrogen bonds [31,32]. Under the influence of shear, enhanced interactions between these groups could have further reduced the intermolecular hydrogen bonds between cellulose chains. However, when DMAc/LiCl-treated cellulose was subjected to mechanochemical shear, the $3400 \mathrm{~cm}^{-1}$ absorption peak has exhibited only a minor shift to $3386 \mathrm{~cm}^{-1}$ as shown in figure $1 \mathrm{~d}$. This minor shift for the NBRassisted mechanochemically treated MCC could be attributed to favourable competitive interactions between NBR and $\mathrm{DMAc} / \mathrm{LiCl}$ which could be higher than the interactions between NBR and cellulose or cellulose and DMAc/LiCl. As a result, changes in intermolecular hydrogen bonds for this sample could have been relatively less which accounts for the minor shift to $3386 \mathrm{~cm}^{-1}$ for the mechanochemically treated sample when compared to the absorption at $3351 \mathrm{~cm}^{-1}$ for the mechanically treated sample.

FTIR spectra of mechanically and mechanochemically treated MCC samples assisted by EPDM rubber together with the untreated and DMAc/LiCl-treated samples are shown in figure $2 \mathrm{a}$ and $\mathrm{b}$. The $\mathrm{OH}$ stretching vibration peak shifts from 3400 to $3410 \mathrm{~cm}^{-1}$ in this sample due to the absence of any interactions between cellulose and the non-polar EPDM rubber even under the influence of mechanical shear. In the absence of such interactions, EPDM rubber can assist stretching and orientation in cellulose chains in the shear flow field, thereby resulting in the formation of free hydroxyl groups for which absorption occurs at $3410 \mathrm{~cm}^{-1}$ as shown in figure 2c. This is in contrast with the sample in figure $1 \mathrm{c}$ wherein polar interactions between NBR and cellulose predominate over orientation effects in the shear flow field. However, it is interesting to note that when $\mathrm{DMAc} / \mathrm{LiCl}$-treated $\mathrm{MCC}$ is subjected to EPDM rubber-assisted mechanochemical shear, a

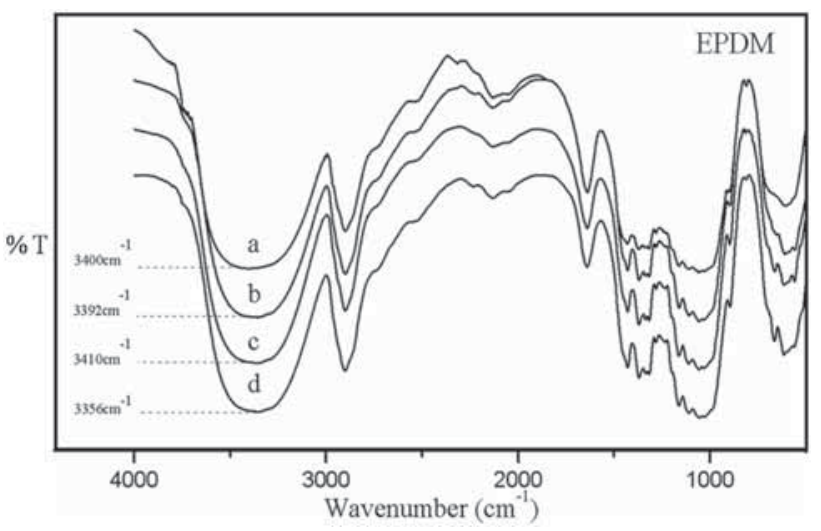

Figure 2. FTIR spectra of (a) untreated MCC, (b) DMAc/LiCltreated MCC, (c) untreated MCC subjected to EPDM-assisted mechanical shear and (d) DMAc/LiCl-treated MCC subjected to mechanochemical shear.

significant shift occurs in the $\mathrm{OH}$ stretching peak from 3410 to $3356 \mathrm{~cm}^{-1}$ as evident from figure $2 \mathrm{~d}$. This can be attributed to the extensive interactions between cellulose molecules and DMAc/LiCl under the influence of EPDM-assisted shear. EPDM rubber provides the necessary shear flow field without itself involving in any competitive chemical interactions with MCC unlike NBR. Shear-induced orientation in this case enhances proximity between cellulose hydroxyl groups and $\mathrm{DMAc} / \mathrm{LiCl}$, thereby significantly increasing the interactions between them. As a result, reduction in intermolecular hydrogen bonds between cellulose chains is evident as reflected in the shift in the peak position from 3410 to $3356 \mathrm{~cm}^{-1}$.

\subsection{Crystallinity of cellulose subjected to mechanical and mechanochemical shear}

XRD studies have been carried out to understand the effect of mechanical and mechanochemical shear on the crystallinity of MCC samples. XRD patterns of samples subjected to NBR-assisted shear are shown in figure 3 along with that of untreated and DMAc/LiCl-treated samples for comparison. XRD of untreated and DMAc/LiCl-treated MCC samples (figure $3 \mathrm{a}$ and $\mathrm{b}$ ) exhibit sharp crystalline peaks at the characteristic $2 \theta$ value of $22^{\circ}$ corresponding to the (002) plane. It is to be noted that the peak intensity or position of DMAc/LiCltreated MCC (figure $3 \mathrm{~b}$ ) is almost similar to that of untreated MCC (figure 3a). However, XRD of the untreated MCC sample subjected to NBR-assisted mechanical shear clearly shows a significant drop in peak intensity as evident from figure $3 \mathrm{c}$. The $\mathrm{DMAc} / \mathrm{LiCl}$ pre-treated sample under similar conditions has not shown any change in its peak intensity as shown in figure $3 \mathrm{~d}$. The significant drop in peak intensity for NBR-assisted mechanically treated MCC, shown in figure 3c, can be attributed to enhanced solid-state chemical interactions between nitrile groups of NBR and the OH groups of cellulose involved in the intermolecular hydrogen bonds. As a result, intermolecular hydrogen bonds between hydroxyl of 


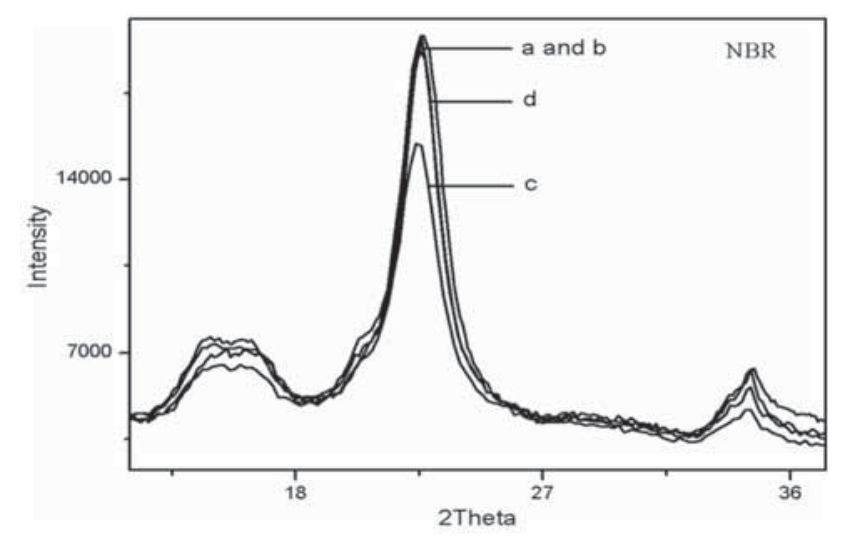

Figure 3. XRD patterns of (a) untreated MCC, (b) DMAc/LiCltreated MCC, (c) untreated MCC subjected to NBR-assisted mechanical and (d) DMAc/LiCl-treated MCC subjected to mechanochemical shear.

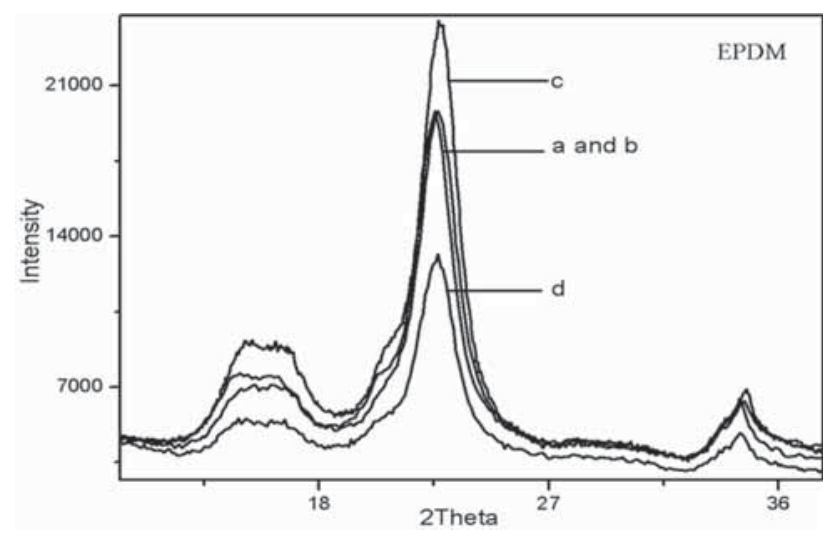

Figure 4. XRD patterns of (a) untreated MCC, (b) DMAc/LiCltreated MCC, (c) untreated MCC subjected to EPDM rubberassisted mechanical and (d) DMAc/LiCl-treated MCC subjected to mechanochemical shear.

cellulose chains have decreased which could have reduced the crystallinity of this sample. On the other hand, when DMAc/LiCl pre-treated MCC is subjected to NBR-assisted mechanochemical shear, competitive preferential interactions between NBR and DMAc/LiCl limit not only the interactions between cellulose and $\mathrm{DMAc} / \mathrm{LiCl}$, but also between cellulose and NBR. This has left the crystallinity of this sample relatively unaffected as evident from figure $3 \mathrm{~d}$.

XRD patterns of untreated and DMAc/LiCl-treated MCC samples subjected to EPDM rubber-assisted mechanical and mechanochemical shear show a contrary trend as shown in figure 4. When EPDM rubber is used to assist mechanical shear, the shear flow region provided by the rubber, in the absence of any chemical interactions, can only induce extensive orientation in cellulose chains which could have increased its crystallinity. This is evident from the sharp increase in the maximum peak intensity of this sample at the $2 \theta$ value of $22^{\circ}$ as shown in figure $4 \mathrm{c}$. However, with DMAc/LiCl-treated MCC during mechanochemical shear, EPDM rubber neither interacts with cellulose nor with $\mathrm{DMAc} / \mathrm{LiCl}$, but only facilitates necessary shear and proximity for extensive interactions between the absorbed $\mathrm{DMAc} / \mathrm{LiCl}$ and hydroxyl groups of MCC in the solid state. These extensive interactions are capable of effectively disrupting the intermolecular hydrogen bonds between MCC chains which could be attributed to the significant drop in peak intensity for this sample, as evident from figure $4 \mathrm{~d}$. As a result, the crystalline index has decreased significantly as reported earlier [28]. The crystalline index data for all the samples are shown in table 1 .

\subsection{TGA of cellulose subjected to shear}

TGA has been used to compare the thermal stability of untreated MCC with that of mechanically and mechanochemically treated MCC samples and the thermograms are shown in figures 5 and 6 . For all the samples, the initial mass loss up to $150^{\circ} \mathrm{C}$ is due to the evaporation of absorbed moisture. For the untreated $\mathrm{MCC}$, second stage degradation starts at $300^{\circ} \mathrm{C}$ at a peak degradation temperature of $340^{\circ} \mathrm{C}$ as shown in the thermogram of figure 5a. For the mechanically treated MCC

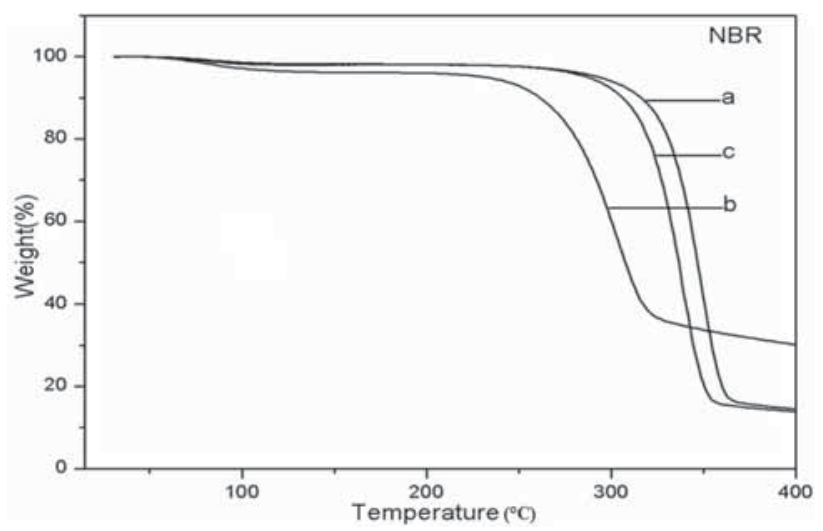

Figure 5. TGA curves of (a) untreated MCC, (b) untreated MCC subjected to NBR-assisted mechanical shear and (c) DMAc/LiCltreated MCC subjected to mechanochemical shear.

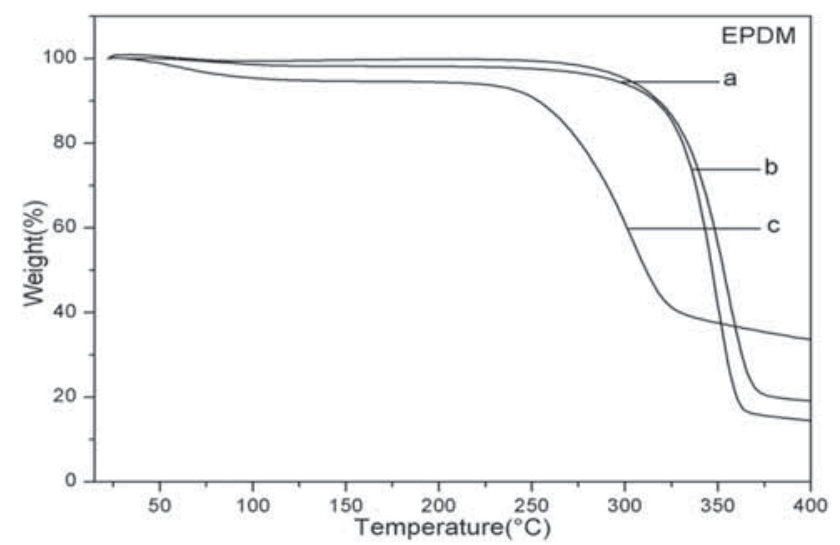

Figure 6. TGA curves of (a) untreated MCC, (b) untreated MCC subjected to EPDM rubber-assisted mechanical and (c) DMAc/LiCltreated MCC subjected to mechanochemical shear. 
using NBR as the shear transfer medium, the second stage degradation starts at a significantly lower temperature of $225^{\circ} \mathrm{C}$ with a peak degradation temperature at $300^{\circ} \mathrm{C}$ as shown in the thermogram of figure $5 \mathrm{~b}$. This significant decrease in both onset and peak degradation temperatures indicates that mechanical treatment using NBR has resulted in more amorphous content in MCC with a corresponding reduction in its crystalline content which correlates well with the XRD results discussed in the previous section. However, thermal degradation of the mechanochemically treated MCC using NBR, as shown in the thermogram of figure $5 \mathrm{c}$, has exhibited only a small variation from the untreated MCC. The onset and peak degradation temperatures for the mechanochemically treated sample using NBR are observed at relatively high temperatures at 290 and $330^{\circ} \mathrm{C}$, respectively. It is important to note that in the case of NBR-assisted mechanochemical treatment of $\mathrm{MCC}$, preferential interactions between NBR and $\mathrm{DMAc} / \mathrm{LiCl}$ could have reduced the interactions between cellulose and DMAc/LiCl. Crystallinity and thermal stability of this sample therefore, do not differ much from that of untreated MCC as can be seen from the thermogram of figure 5a.

When EPDM rubber is used as the shear transfer medium, the resultant high crystallinity of the mechanically treated MCC sample has shown higher onset and peak degradation temperatures at 300 and $340^{\circ} \mathrm{C}$, respectively (thermogram of figure $6 \mathrm{~b}$ ) and similar to that of untreated MCC (thermogram of figure 6a). Mechanochemical treatment of the DMAc/LiCl-treated MCC sample using EPDM rubber has, however, shown significantly lower onset and peak degradation temperatures at 240 and $300^{\circ} \mathrm{C}$, respectively, as shown in the thermogram of figure $6 \mathrm{c}$ which could be attributed to the reduced crystallinity and higher amorphous content of this sample. Degradation of the cellulose sample with more amorphous content starts at lower temperatures than the sample with higher crystalline content. In this sample, interactions between $\mathrm{DMAc} / \mathrm{LiCl}$ and hydroxyl groups of cellulose are facilitated to the maximum extent during shear which has resulted in more amorphous content and as a consequence, reduced the thermal stability in this sample.

\subsection{Swelling of cellulose subjected to mechanical and mechanochemical shear}

Dissolution of cellulose in organic solvents is difficult because of the presence of strong hydrogen bonded networks and the associated high crystallinity. For dissolution to occur in organic solvents, disruption of the hydrogen bond network and the crystalline order in cellulose are therefore essential. The effects of mechanical and mechanochemical treatments on the swelling and dissolution behaviour of MCC are reported in this section. Samples of untreated MCC, DMAc/LiCl-treated MCC and MCC subjected to the elastomer (NBR and EPDM rubbers)-assisted mechanical and mechanochemical treatments have been subjected to swelling and/or dissolution in several organic media. Even

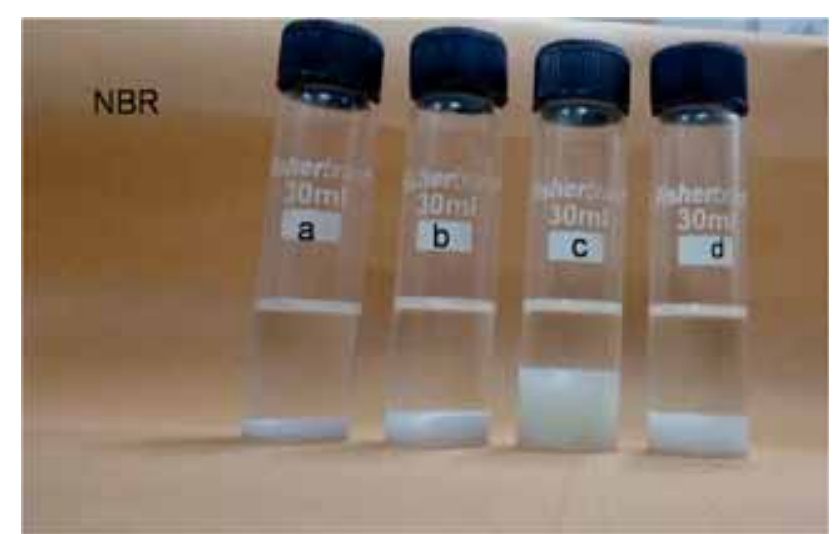

Figure 7. Swellings of (a) untreated MCC, (b) DMAc/LiCltreated MCC, (c) untreated MCC subjected to NBR-assisted mechanical and (d) DMAc/LiCl-treated MCC subjected to mechanochemical shear.

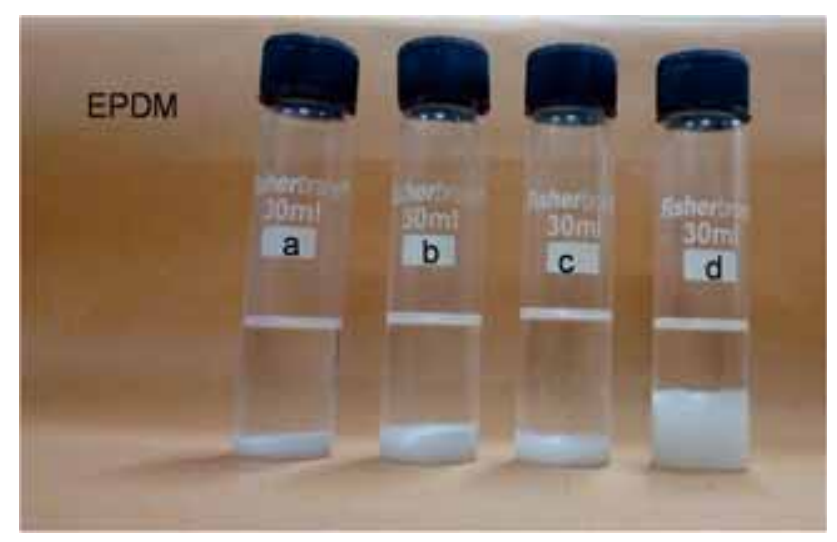

Figure 8. Swellings of (a) untreated MCC, (b) DMAc/LiCltreated MCC, (c) untreated MCC subjected to EPDM rubberassisted mechanical and (d) DMAc/LiCl-treated MCC subjected to mechanochemical shear.

with mechanical and mechanochemical treatments, neither dissolution nor swelling of MCC samples could be observed in polar solvents like THF, DMF and DMAc. Hence, the effectiveness of these treatments on swelling and dissolution of $\mathrm{MCC}$ in DMAc/LiCl solution is investigated and the results, in visual forms, are shown in figures 7 and 8 .

Figure $7 \mathrm{a}$ and $\mathrm{b}$ shows the swelling behaviour of untreated and $\mathrm{DMAc} / \mathrm{LiCl}$-treated $\mathrm{MCC}$ respectively in $\mathrm{DMAc} / \mathrm{LiCl}$ solution at the end of $48 \mathrm{~h}$. It can be seen that in the absence of shear, but with $\mathrm{DMAc} / \mathrm{LiCl}$ treatment alone, the sample (figure 7b) exhibits only a small increase in swelling when compared to that of the untreated sample (figure 7a). But, when subjected to NBR-assisted mechanical shear, the sample has exhibited a significant increase in swelling as shown in figure 7c. Higher swelling of this sample could be directly attributed to the relatively opened up amorphous structure of cellulose. Closer interactions between nitrile groups and cellulose in the intensive shear flow region effectively translate the enormous shear stresses onto 
cellulose which in turn could have significantly reduced the intermolecular hydrogen bonding between MCC chains and crystallinity in the sample thereby resulting in its higher swelling. The mechanochemically treated MCC sample, assisted by NBR (figure 7d), however, does not exhibit any significant swelling in $\mathrm{DMAc} / \mathrm{LiCl}$ when compared to the mechanically treated sample as shown in figure $7 \mathrm{c}$. In the former, more polar interactions between nitrile groups and $\mathrm{DMAc} / \mathrm{LiCl}$ occur during shear than interactions between cellulose and $\mathrm{DMAc} / \mathrm{LiCl}$ or between cellulose and NBR and therefore, the hydrogen bond network in MCC is not disturbed much. The preferential interaction between nitrile groups and $\mathrm{DMAc} / \mathrm{LiCl}$ reduces the interaction between cellulose and $\mathrm{DMAc} / \mathrm{LiCl}$ thereby leaving crystallinity unaffected. Hence, only limited swelling is observed in this sample.

The trend in swelling of MCC in $\mathrm{DMAc} / \mathrm{LiCl}$ is reversed when the samples are subjected to mechanical and mechanochemical shear using EPDM rubber. Due to their difference in polarity, chemical interactions between cellulose and EPDM rubber is completely absent. In the absence of such interactions, the shear flow field has facilitated extensive orientation in cellulose chains which increases its crystallinity. This is the reason for the reduced swelling of this sample as evident from figure 8c. When DMAc/LiCl-treated MCC is subjected to EPDM-assisted mechanochemical action, however, extensive interactions between the hydroxyl groups of cellulose and $\mathrm{DMAc} / \mathrm{LiCl}$ are facilitated in the shear region even in the solid state. Such close solid-state interactions between cellulose and $\mathrm{DMAc} / \mathrm{LiCl}$ are possible only under the influence of shear imposed by a non-polar medium such as EPDM rubber. It is important to note that such close interactions between cellulose and $\mathrm{DMAc} / \mathrm{LiCl}$ are either limited or does not exist in the absence of a shear field as can be seen from the limited or poor swelling of the DMAc/LiCl-treated MCC sample shown in figure $8 \mathrm{~b}$. Thus, the combination of chemical interactions and mechanical shear has been found to be effective in reducing the hydrogen bond network and crystallinity in MCC which in turn facilitates extensive swelling of this cellulose sample as evident from figure $8 \mathrm{~d}$. Therefore, decrystallization of MCC has been realized through enhanced solid-state chemical interactions between cellulose and $\mathrm{DMAc} / \mathrm{LiCl}$ under the influence of EPDM rubber-assisted shear.

\subsection{Surface morphological characteristics of regenerated cellulose}

Cellulose samples after swelling and partial dissolution in DMAc/LiCl have been regenerated and the dried samples are analysed using SEM to understand the effect of mechanical and mechanochemical treatments on their surface morphology and the micrographs are shown in figure 9 . Regenerated samples from untreated and DMAc/LiCl-treated MCC, in the absence of any shear, have not shown much variation in their morphological features as observed in figure $9 \mathrm{a}$ and $\mathrm{b}$. Merely mixing $\mathrm{MCC}$ with $\mathrm{DMAc} / \mathrm{LiCl}$ is not sufficient enough to favour any chemical interactions between
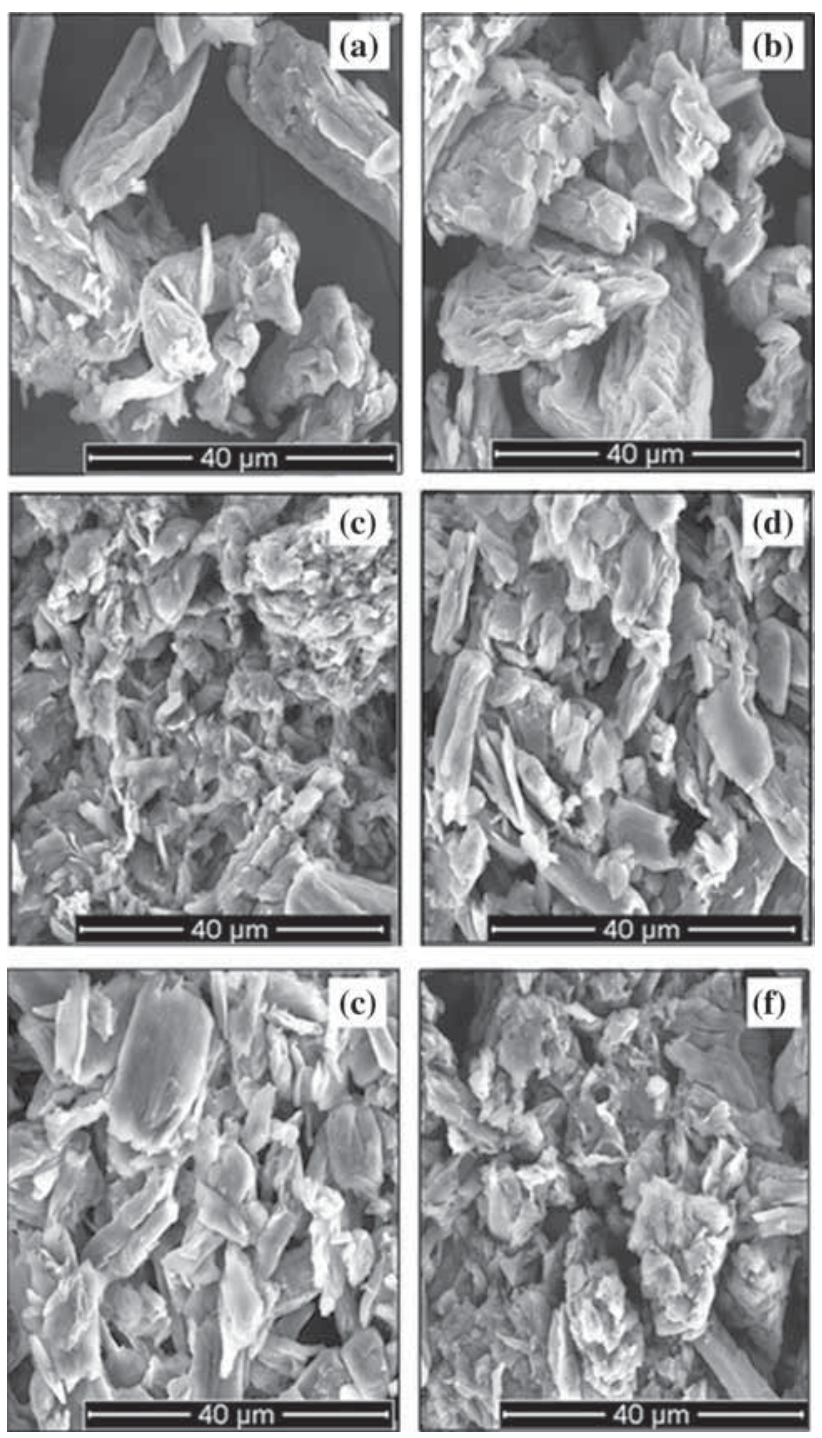

Figure 9. SEM micrographs of regenerated MCC from (a) untreated MCC, (b) DMAc/LiCl-treated MCC, (c) untreated MCC subjected to NBR-assisted mechanical shear, (d) DMAc/LiCltreated MCC subjected to mechanochemical shear, (e) untreated MCC subjected to EPDM rubber-assisted mechanical shear and (f) $\mathrm{DMAc} / \mathrm{LiCl}$-treated MCC subjected to mechanochemical shear (for the above samples, the magnification is $1000 \times$ ).

the two, though the latter is a solvent for the former. However, figure $9 \mathrm{c}$ shows that the regenerated sample from mechanically treated MCC assisted by NBR has shown a reduction in the particle size and a significantly opened up amorphous morphology when compared to untreated MCC shown in figure 9a. More amorphous morphology of this sample could be the reason for its increased swelling in $\mathrm{DMAc} / \mathrm{LiCl}$. This is further supported by the TGA thermogram of this sample in which the onset and peak degradation temperatures are significantly lower as shown in figure $5 \mathrm{~b}$. But, the morphology of regenerated MCC obtained from NBR-assisted mechanochemical shear (figure 9d) does not differ 
significantly from that of the untreated sample which has also been reflected in its XRD, TGA and swelling characteristics.

SEM images of regenerated cellulose samples obtained from mechanically and mechanochemically treated MCC assisted by EPDM rubber are shown in figure 9e and $\mathrm{f}$, respectively. The regenerated sample obtained from mechanical shear alone has shown more broken fibrous morphology due to the extensive orientation of cellulose in the shear region which has resulted in purely mechanical breakdown of cellulose as shown in figure 9e. This is further evident from the higher crystallinity and lesser swelling characteristics observed for this sample. The regenerated MCC sample, obtained from DMAc/LiCl-treated MCC subjected to EPDM rubber-assisted mechanochemical shear, however, has shown more amorphous like surface morphology as evident from figure 9f. It is evident that EPDM rubber-assisted shear has facilitated extensive solid-state interactions between $\mathrm{DMAc} / \mathrm{LiCl}$ and cellulose which has substantially reduced the intermolecular hydrogen bond networks in MCC. This is clearly seen from the significant reduction in the crystallinity and higher swelling of this sample. All these observations strongly indicate solid-state decrystallization in MCC under the influence of EPDM rubber-assisted mechanochemical shear.

\section{Conclusions}

Decrystallization of MCC subjected to elastomer-assisted mechanical and mechanochemical shear has been achieved using NBR and EPDM rubber as shear transfer media. The objective of this work has been to facilitate solidstate interactions between hydroxyl groups of cellulose and $\mathrm{DMAc} / \mathrm{LiCl}$ under the influence of elastomer-assisted shear. It has been found that polarity of the elastomer used for mechanical and mechanochemical treatments influenced the morphology, crystallinity, thermal stability and swelling characteristics of untreated and DMAc/LiCl-treated MCC. In the absence of elastomer-assisted shear, difference in crystallinity between untreated and DMAc/LiCl-treated MCC has been found to be negligible. The FTIR results have revealed changes in stretching vibrations of cellulose hydroxyl groups under the influence of NBR-assisted mechanical shear and EPDM rubber-assisted mechanochemical shear. XRD patterns and crystalline index data have also indicated reduction in crystallinity of MCC samples subjected to NBR-assisted mechanical shear and EPDM rubber-assisted mechanochemical shear. Reduction in crystalline content has also been confirmed using the TGA results. TGA thermograms have indicated lower onset and peak degradation temperatures for MCC samples with reduced crystallinity. Higher swelling and more amorphous like surface morphology have been observed for regenerated MCC samples subjected to NBR-assisted mechanical shear and EPDM rubber-assisted mechanochemical shear. Hence, decrystallization of cellulose through enhanced solid-state interactions between hydroxyl groups and DMAc/LiCl is possible by employing EPDM rubber-assisted mechanochemical shear.

\section{References}

[1] Altaner C M, Thomas L H, Fernandes A N and Jarvis M C 2014 Biomacromolecules 15791

[2] Soheilmoghaddam M, Wahit M U and Akos N I 2013 Mater. Lett. 111221

[3] Gupta K M and Jiang J 2015 Chem. Eng. Sci. 121180

[4] Ohno E and Miyafuji H J 2013 J. Wood Sci. 59221

[5] Swatloski R P, Spear S K, Holbrey J D and Rogers R D 2002 J. Am. Chem. Soc. 1244974

[6] Zhu S, Wu Y, Chen Q, Yu Z, Wang C, Jin S et al 2006 Green Chem. 8325

[7] Benoit J C, Newman R H and Staiger M P 2007 Cellulose 14 311

[8] Isik M, Sardon H and Mecerreyes D 2014 Int. J. Mol. Sci. 15 11922

[9] Potthast A, Rosenau T, Sixta H and Kosma P 2002 Tetrahedron Lett. 437757

[10] Striegel A 1997 Carbohydr. Polym. 34267

[11] Stryuk S, Eckelt J and Wolf B A 2005 Cellulose 12145

[12] Zhang C, Liu R, Xiang J, Kang H, Liu Z and Huang Y 2014 J. Phys. Chem. B 1189507

[13] Khan A S, Man Z, Bustam M A, Kait C F, Khan M I, Muhammad N et al 2016 Waste Biomass Valori. 7571

[14] Tonoli G H, Holtman K M, Glenn G, Fonseca A S, Wood D, Williams T et al 2016 Cellulose 231239

[15] Zhang W, Liang M and Lu C 2007 Cellulose 14447

[16] Zhang W, Yang X, Li C, Liang M, Lu C and Deng Y 2011 Carbohydr. Polym. 83257

[17] Jain M and Pradhan M K 2016 Int. J. Plast. Technol. 20378

[18] Kalia S, Kaith B S and Kaur I 2009 Polym. Eng. Sci. 491253

[19] O’Connor J E 1977 Rubber Chem. Technol. 50945

[20] Visakh P M, Thomas S, Oksman K and Mathew A P 2012 BioResources 72156

[21] Zhou Y, Fan M, Chen L and Zhuang J 2015 Compos. Part B 76 180

[22] Cao X, Xu C, Liu Y and Chen Y 2013 Carbohydr. Polym. 92 69

[23] Iyer K A, Schueneman G T and Torkelson J M 2015 Polymer 56 464

[24] Parambath Kanoth B, Claudino M, Johansson M, Berglund L A and Zhou Q 2015 Appl. Mater. Interfaces 716303

[25] Sajithkumar K J, Visakh P M and Ramasamy E V 2016 Waste Biomass Valori. 71227

[26] Lavanya R, Clara I and Natchimuthu N 2017 J. Appl. Polym. Sci. 13444809

[27] Segal L, Creely J J, Martin A E and Conrad C M 1959 Text. Res. J. 29786

[28] Xiong R, Zhang X, Tian D, Zhou Z and Lu C 2012 Cellulose 19 1189

[29] Salmen L and Bergstrom E 2009 Cellulose 16975

[30] Hinterstoisser B and Salmen L 1999 Cellulose 6251

[31] Cao X, Xu C, Wang Y, Liu Y, Liu Y and Chen Y 2013 Polym. Test. 32819

[32] Chen Y, Zhang Y, Xu C and Cao X 2015 Carbohydr. Polym. 130 149 